\title{
Mathematical Modelling of Coronavirus disease (COVID-19) Outbreak in India using Logistic Growth and SIR Models
}

\section{Joby Mackolil}

CHRIST (Deemed to be University) https://orcid.org/0000-0003-4829-5381

Basavarajappa Mahanthesh ( $\nabla$ mahanthesh.b@christuniversity.in )

CHRIST (Deemed to be University) https://orcid.org/0000-0003-2481-3842

Keywords: COVID-19, Novel Corona virus, Epidemic, Mathematical modelling, Logistic growth model, SIR model

Posted Date: May 28th, 2020

DOI: https://doi.org/10.21203/rs.3.rs-32142/v1

License: (c) (i) This work is licensed under a Creative Commons Attribution 4.0 International License.

Read Full License 


\title{
Mathematical Modelling of Coronavirus disease (COVID-19) Outbreak in India using Logistic Growth and SIR Models
}

\author{
Joby Mackolil and Basavarajappa Mahanthesh* \\ Department of Mathematics, CHRIST (Deemed to be University), Bengaluru-560029, India.
}

\section{*Email of corresponding author: mahanthesh.b@christuniversity.in}

\begin{abstract}
The mathematical modelling of the Coronavirus disease (COVID-19) outbreak in India is done by using the logistic growth model and the Susceptible-Infectious-Recovered (SIR) framework. Karnataka, Kerala and Maharashtra, three states of India, are selected based on the pattern of the disease spread and the prominence in being affected in India. The parameters of the models are estimated by utilizing real-time data. The models predict the ending of the pandemic in these states and estimate the number of people that would be affected under the prevailing conditions. The models classify the pandemic into five stages based on the nature of the infection growth rate. According to the estimates of the models it can be concluded that Kerala is in a stable situation whereas the pandemic is still growing in Karnataka and Maharashtra. The infection rate of Karnataka and Kerala are lesser than 5\% and reveal a downward trend. On the other hand, the infection rate and the high predicted number of infectives in Maharashtra calls for more preventive measures to be imposed in Maharashtra to control the disease spread.
\end{abstract}

Keywords: COVID-19; Novel Corona virus; Epidemic; Mathematical modelling; Logistic growth model; SIR model. 


\section{Introduction}

The novel Corona virus disease-2019 (COVID-19) is a deadly infectious disease which was first reported in Wuhan, China by the end of the year 2019. Later, it spread across continents leaving only a few countries unaffected. The large spread and increasing number of deaths made the World Health Organization (WHO) to declare it as a pandemic on 11 March 2020 [1]. The governments of the affected countries have imposed various preventive measures including lockdown, seal down, social distancing, quarantining infected and susceptible individuals, promotion of self-hygiene measures and usage of masks. Recently, Fang et al. [2] reported that the governmental interventions proved to be effective in containing the infection rate.

In India, the pandemic was first reported on 30 January 2020 in Thrissur, a district of Kerala for three individuals who had come from Wuhan. In March, the number of infected individuals multiplied due to individuals who had travelled to India from various affected countries. Initially, the government imposed preventive measures including thermal screening of passengers arriving at the airport, shut down of educational institutions and suspending tourist visas. Further, on 24 March, the government imposed a nationwide lock down as a major preventive measure to contain the spread of the virus. This restriction on the human mobility was reported to be effective for various infectious diseases [3, 4]. The Indian context of the disease spread and the impact of the public health interventions were mathematically modelled by Mandal et al. [5].

During these hard times of the pandemic, researchers from various disciplines conduct intensive research to analyze it and to curb its ill-effects [6-8]. Apart from virologic and experimental studies, mathematical and statistical explorations are also important for the decision-makers and policy makers to evaluate the current situation and to take the necessary 
actions for the future. These models also provide an estimate of the ending time and possible number of people that can be infected by this pandemic. The logistic growth model is an efficient technique for epidemic forecasting. Chowell et al. [9] used this model to estimate the transmission of the Ebola virus. They concluded that the predictions from the model were inconsistent in the initial period of the epidemic. The model was also adopted by Pell et al. [10] to estimate the final size and the peak time of the infection. The SIR model (compartmental disease model) is a mathematical method to describe the epidemic growth through a system of time dependent differential equations. The differential equations are based on the compartments into which the population is divided. The SIR model and various modified SIR models were widely used by researchers to model Ebola [11] and AIDS [12]. Recently, such models were used to model the coronavirus epidemic spreading. Khrapov and Loginova [13] utilized the modified SIR model for the analyzing the dynamics of the COVID-19 pandemic in China. In this direction, the modified SEIR model was used by Berger et al. [14] to include the effects of quarantining and infection testing.

Aggravated by the findings of the above explorations, the present article aims at studying the dynamics of the novel Corona virus (COVID-19) in India. The mathematical modelling of the COVID-19 outbreak is done for three states of India, namely Karnataka, Kerala and Maharashtra due to the significant difference in the outbreak pattern. The logistic growth model and the SIR model are used to analyze the situation in these states and the results of the models are compared. The simulations are carried out with the parameters that are estimated from the real time data by utilizing MATLAB. The data from 9 March 2020 to 9 May 2020 are used for the modelling. The final size of the pandemic in the states is estimated and the date when the pandemic becomes stable is also predicted. 


\section{Mathematical models}

\subsection{Logistic growth model}

The population growth can be modelled using the logistic growth model which is a sigmoid curve. Pierre-Francois Verhulst pioneered the usage of logistic growth models for biological systems.

$$
\frac{d \mathcal{C}}{d t}=r \mathcal{C}\left(1-\frac{\mathcal{C}}{K}\right)
$$

where, $\mathcal{C}$-accumulated number of cases,

$r$-infection rate, $t$-time and

$K$ is the final epidemic size.

If the initial number of cases is given by $\left.\mathcal{C}\right|_{t=0}=\mathcal{C}_{0}$, then the solution (1) is

$$
\begin{aligned}
& \mathcal{C}=\frac{K \mathcal{C}_{0} e^{r t}}{K+\mathcal{C}_{0}\left(e^{r t}-1\right)} \\
& \text { i.e., } \mathcal{C}=\frac{K}{1+A e^{-r t}} \text { where, } A=\left(\frac{K-\mathcal{C}_{0}}{\mathcal{C}_{0}}\right) .
\end{aligned}
$$

For estimating the maximum number of affected people, the cases follow the Weibull function. Further, the highest growth rate occurs at the time $t_{\max }=\frac{\ln A}{r}$. At $t_{\text {max }}$, the number of cases is $\frac{K}{2}$. The epidemy is modelled using five stages based on the time $t_{\text {max }}$. This is based on the nature of the growth rate. The five stages are as follows:

\section{Stage 1: Slow growth stage}


The spread of any infectious disease is slow initially which is accounted in this stage. This is the exponential growth phase of the logistic growth model. The growth at the time $t<t_{\max }-$ $\frac{2}{r}$ is considered in this stage.

\section{Stage 2: Accelerating growth stage}

Here the spread of the disease spreads largely in the population. At this stage the number of positive cases multiplies several folds. This accelerating phase is at the time $t_{\max }-\frac{2}{r}<t<$ $t_{\max }$

\section{Stage 3: Decelerating growth stage}

At this stage the spread of the disease decelerates due to various preventive measures taken. The spread of the disease is controlled and lesser than that of Stage 2. The decelerating growth is at the time $t_{\max }<t<t_{\max }+\frac{2}{r}$.

\section{Stage 4: Transition stage}

This is the stage when the disease growth rate slows down. At this stage the epidemy is said to be under control. The preventive measures taken prove to be effective. The time duration of this stage is $t_{\max }+\frac{2}{r}<t<2 t_{\max }$.

\section{Stage 5: Steady stage}

This stage can be regarded as the culminating stage of the epidemy. There is no growth in the disease spread. This stage is at the time $t>2 t_{\max }$.

\subsection{SIR model}

The Susceptible-Infected-Recovered (SIR) model is a mathematical model also known as compartmental disease model to describe the disease spread in a population. Here, the 
considered population belongs to any one of the three compartments: Susceptible, Infected or Recovered as shown in Fig. 1.

Susceptible are the individuals without immunity to the disease, hence they can be infected. Susceptible individuals can move to the next compartment (Infectious) through the interaction with infectious beings. Infectious beings are infected with the disease and they can transmit the disease to the susceptible individuals. By recovering from the illness, they can move to the next compartment called Recovered. Now, recovered beings can no longer develop the infection as they gained immunity via prior exposure. As beings can move among compartments, the number of members in each compartment varies with time. This model was first constructed by Kermack and McKendrick [15], it comprises of following set of time related nonlinear ordinary differential equations to simulate the growth of a disease.

$$
\begin{aligned}
& \frac{d S(t)}{d t}=-\frac{\beta}{N} S(t) I(t), \\
& \frac{d I(t)}{d t}=\frac{\beta}{N} S(t) I(t),-\gamma I(t), \\
& \frac{d R(t)}{d t}=\gamma I(t),
\end{aligned}
$$

where, $S(t)$ is the number of susceptible individuals,

$I(t)$ is the number of infected individuals

$R(t)$ is the number of recovered individuals

$N$ is the considered constant population size involved in the disease

$\beta$ is the contact rate of the disease

$\gamma$ is the mean recovery/removal rate.

The initial conditions are $S(t=0), I(t=0), R(t=0) \geq 0$. Let it be respectively $S_{0}, I_{0}$ and $R_{0}$. 
The following suppositions are involved in the model:

- The population is considered to be unvarying during the phase of modelling.

- All the infected beings have an equal chance to be recovered.

- Secondary waves of infections and any other unusual outbreak of the infection are not considered in these models.

- The real time data of the officially reported positive cases are used for the models.

Solving the equations one can get,

$$
S(t)=S_{0} e^{-\frac{\beta}{N \gamma}\left(R(t)-R_{0}\right)}
$$

To find the total number of susceptible individuals at the end of the disease spread, the limit to $\infty$ is taken to get:

$$
S_{\infty}=S_{0} e^{-\frac{\beta}{N \gamma}\left(R_{\infty}-R_{0}\right)}
$$

where, $R_{\infty}$ is the final number of recovered individuals. In order to estimate the model parameters and $S_{0}$ with the available real time data of the number of positive cases (denoted by $C_{i}$ for each day $i$ ), the initial values are taken to be $I_{0}=C_{1}$ and $R_{0}=0$. Now, the parameters are found such that the error sum of square of the predicted and the actual number of cases is minimum.

\section{Methodology}

The number of positive novel corona virus (COVID-19) cases in Karnataka, Kerala and Maharashtra from 9 March 2020 to 9 May 2020 were recorded. The data source was based on the daily reports from the respective health departments of the states. These states were selected due to the significant difference in the disease spread patterns. This data was used to estimate the parameters for the logistic growth model and the SIR model. The models and the 
visualizations are done using the fitvirus program developed by Batista $[16,17]$ using MATLAB.

\section{Interpretations of the Results}

\section{(a) Logistic growth model}

Table 1 summarizes the estimated parameters for the logistic growth model. Within two months, Kerala reached the steady stage (Stage 5) whereas Maharashtra and Karnataka are in the decelerating growth stage (Stage 3). Though Karnataka and Maharashtra are in the same stage, the intensity of the disease spread is different. The logistic growth model for the three states is displayed in Figs 2-4.

Table 1: Estimated logistic growth model summary.

\begin{tabular}{|l|c|c|c|}
\hline \multicolumn{1}{|c|}{ Parameter } & Kerala & Karnataka & Maharashtra \\
\hline Epidemic stage & Stage 5 & Stage 3 & Stage 3 \\
\hline Number of cases on 9 May 2020 & 505 & 794 & 20228 \\
\hline Epidemic size (cases) & 485 & 1009 & 43993 \\
\hline Duration of accelerating growth (days) & 29 & 44 & 33 \\
\hline date of $t_{\max }$ & $1-04-2020$ & $25-04-2020$ & $08-05-2020$ \\
\hline End of transition stage & $24-04-2020$ & $11-06-2020$ & $07-07-2020$ \\
\hline Total $R^{2}$ & $98.4 \%$ & $99.5 \%$ & $99.9 \%$ \\
\hline
\end{tabular}

Considering that the same situations prevail in the states and the present growth rates, the estimated maximum number of people who will be infected in Maharashtra, Karnataka and Kerala are 43993, 1009 and 485 respectively. It is to be noted that the prediction of Kerala is lesser underestimated. This can be due to secondary disease infection waves or migration. This is visualized in Figs 5-7. The duration of the accelerating growth stage and the date corresponding to $t_{\max }$ are tabulated in Table 1 . The end of the transition stage can be regarded as the stage at which the disease spread is under control. It is interesting to note that the estimated end of the transition stage for Maharashtra is by 7 July while it is by 24 April in 
Kerala and 11 June in Karnataka. The high values of the coefficient of determination $\left(R^{2}\right)$ suggest the accuracy of the fitted models.

\section{(b) SIR Model}

The estimated parameters for the SIR model of the three states are given in Table 2. The contact rate quantifies the mean number of contacts per infected individual per day. It is seen that the contact rate is the highest for Maharashtra. The exclusion of the infected population by immunization, death, quarantining or isolation is quantified using the removal rate $(\gamma)$. A high removal rate $(\gamma=0.914)$ is estimated for Maharashtra. The estimated number of individuals who are likely to be involved in the coronavirus outbreak in each state is 487 (Kerala), 6626 (Karnataka) and 213604 (Maharashtra). In the aforementioned estimated numbers, the estimated number of infections in each state is found to be 449 (Kerala), 1177 (Karnataka) and 46678 (Maharashtra). The remaining individuals are the estimated number of susceptible individuals. Time between contacts is the reciprocal of the contact rate and the time between contacts is the reciprocal of the removal rate. The respective values of each state are recorded in Table 2 . The basic reproduction number $\left(\mathcal{R}_{0}\right)$ quantifies the expected number of secondary infections by one typical infection in a population that is completely susceptible. Based on the data it is estimated that the $\mathcal{R}_{0}$ of Kerala is the highest among the three states. The high predicted number of cases of Maharashtra indicates that the state must enforce more strict measures to curb the spread of the virus.

Table 2: Estimated susceptible-infected-recovered (SIR) model parameters

\begin{tabular}{|l|l|l|l|}
\hline Parameter & Kerala & Karnataka & Maharashtra \\
\hline Contact rate $(\beta)(/$ day $)$ & 0.312 & 0.836 & 1.031 \\
\hline Removal rate $(\gamma)(/$ day $)$ & 0.114 & 0.76 & 0.914 \\
\hline Final number of cases & 449 & 1177 & 46678 \\
\hline Final number of susceptible & 38 & 5449 & 166926 \\
\hline Involved Population size $(\mathrm{N})$ & 487 & 6626 & 213604 \\
\hline Time between contacts $($ day $)$ & 3.2 & 1.2 & 1 \\
\hline
\end{tabular}




\begin{tabular}{|l|l|l|l|}
\hline Infectious period (day) & 8.8 & 1.3 & 1.1 \\
\hline Basic reproduction number $\left(\mathcal{R}_{0}\right)$ & 2.73684 & 1.1 & 1.128 \\
\hline
\end{tabular}

The SIR model of the states are visualized in Figs. 8-10. The estimated number of infection rate (using the SIR model) is also plotted against the actual number of cases/day. Further, the change in the number of cases is analysed by using the growth rate plot. If the growth rate of the number of new cases reported per day is lesser than $5 \%$, then the outbreak is said to be under control. It can be concluded that the infection growth rate is lesser than $5 \%$ in Kerala and Karnataka but Maharashtra is yet to get control over the infection transmission.

\section{Concluding Remarks}

The COVID-19 outbreak in India was analyzed by comparing the dynamics of the pandemic in Karnataka, Kerala and Maharashtra using the logistic growth and SIR models. It was observed that the states experience a totally different pattern of the disease transmission. Maharashtra and Karnataka are found to be in the decelerating growth stage whereas Kerala is already in the steady stage. The strict and humane prevention measures taken by the government of Kerala may be the reason for the quick control over the pandemic. The size of the pandemic was estimated using the real time data of the daily reported cases. The logistic growth model predicted the maximum number infected individuals in Kerala, Karnataka and Maharashtra to be 485, 1009 and 43993 respectively whereas the SIR model estimated it to be 449, 1177 and 46678 respectively. The complete control of the disease spread is predicted to be by 7 July in Maharashtra while it is by 24 April in Kerala and 11 June in Karnataka. From the SIR model, it is concluded that the infection growth rate is lesser than 5\% in Kerala and Karnataka but Maharashtra experiences is yet to achive that state. The predicted values and dates are based on the prevalant conditions and pattern of disease spread for the considered time period. The high predicted number of infectives in Maharashtra calls for more preventive measures to be taken in the state. 


\section{List of Abbreviations}

COVID-19 - Coronavirus disease 2019;

SIR - Susceptible-Infectious-Recovered;

WHO - World Health Organization.

\section{Declarations}

\section{Availability of data and materials}

The secondary data of the daily reported cases were taken from the daily reports published by the Ministry of Health and Family Welfare (MoHFW), government of India (www.mohfw.gov.in).

\section{Competing interests}

The authors declare that they have no competing interests.

\section{Funding}

There is no funding received for the manuscript.

\section{Authors' Contribution}

Both the authors contributed equally for the planning, analysis and writing of the manuscript. The final version of the manuscript was approved by both the authors.

\section{Acknowledgements}

The authors are thankful to the management of CHRIST (Deemed to be University) for their support.

\section{Authors information}

Both the authors are affiliated to the Department of Mathematics, CHRIST (Deemed to be University), Bangalore, India. 


\section{References}

[1] World Health Organization. "Coronavirus disease 2019”. Available: www.who.int/emergencies/diseases/novel-coronavirus-2019.

[2] Fang, Y., Nie, Y., \& Penny, M. (2020). Transmission dynamics of the COVID-19 outbreak and effectiveness of government interventions: A data-driven analysis. Journal of medical virology.

[3] Bajardi, P., Poletto, C., Ramasco, J. J., Tizzoni, M., Colizza, V., \& Vespignani, A. (2011). Human mobility networks, travel restrictions, and the global spread of 2009 H1N1 pandemic. PloS one, 6(1).

[4] Charu, V., Zeger, S., Gog, J., Bjørnstad, O. N., Kissler, S., Simonsen, L., ... \& Viboud, C. (2017). Human mobility and the spatial transmission of influenza in the United States. PLoS computational biology, 13(2), e1005382.

[5] Mandal, S., Bhatnagar, T., Arinaminpathy, N., Agarwal, A., Chowdhury, A., Murhekar, M., ... \& Sarkar, S. (2020). Prudent public health intervention strategies to control the coronavirus disease 2019 transmission in India: A mathematical model-based approach. Indian J Med Res, 151.

[6] Al-Tawfiq, J. A., Al-Homoud, A. H., \& Memish, Z. A. (2020). Remdesivir as a possible therapeutic option for the COVID-19. Travel medicine and infectious disease.

[7] Wölfel, R., Corman, V. M., Guggemos, W., Seilmaier, M., Zange, S., Müller, M. A., ... \& Hoelscher, M. (2020). Virological assessment of hospitalized patients with COVID-2019. Nature, 1-10.

[8] Hall Jr, D. C., \& Ji, H. F. (2020). A search for medications to treat COVID-19 via in silico molecular docking models of the SARS-CoV-2 spike glycoprotein and 3CL protease. Travel Medicine and Infectious Disease, 101646.

[9] Chowell, G., Simonsen, L., Viboud, C., \& Kuang, Y. (2014). Is West Africa approaching a catastrophic phase or is the 2014 Ebola epidemic slowing down? Different models yield different answers for Liberia. PLoS currents, 6.

[10] Pell, B., Kuang, Y., Viboud, C., \& Chowell, G. (2018). Using phenomenological models for forecasting the 2015 Ebola challenge. Epidemics, 22, 62-70.

[11] Khaleque, A., \& Sen, P. (2017). An empirical analysis of the Ebola outbreak in West Africa. Scientific reports, 7, 42594.

[12] Zakary, O., Larrache, A., Rachik, M., \& Elmouki, I. (2016). Effect of awareness programs and travel-blocking operations in the control of HIV/AIDS outbreaks: a multi-domains SIR model. Advances in Difference Equations, 2016(1), 169. 
[13] Khrapov, P. V., \& Loginova, A. A. (2019). Mathematical modelling of the dynamics of AIDS epidemics development in the world. International Journal of Open Information Technologies, 7(6).

[14] Berger, D. W., Herkenhoff, K. F., \& Mongey, S. (2020). An seir infectious disease model with testing and conditional quarantine (No. w26901). National Bureau of Economic Research.

[15] Kermack, W. O., \& McKendrick, A. G. (1927). A contribution to the mathematical theory of epidemics. Proceedings of the royal society of london. Series A, Containing papers of a mathematical and physical character, 115(772), 700-721.

[16] Batista, M. $\quad$ (2020). $\quad$ fitVirusCOVID19 (https://www.mathworks.com/matlabcentral/fileexchange/74658-fitviruscovid19), MATLAB Central File Exchange.

[17] Batista, $\quad$ M. $\quad$ (2020). fitVirus (https://www.mathworks.com/matlabcentral/fileexchange/74411-fitvirus), MATLAB Central File Exchange. 
Figures

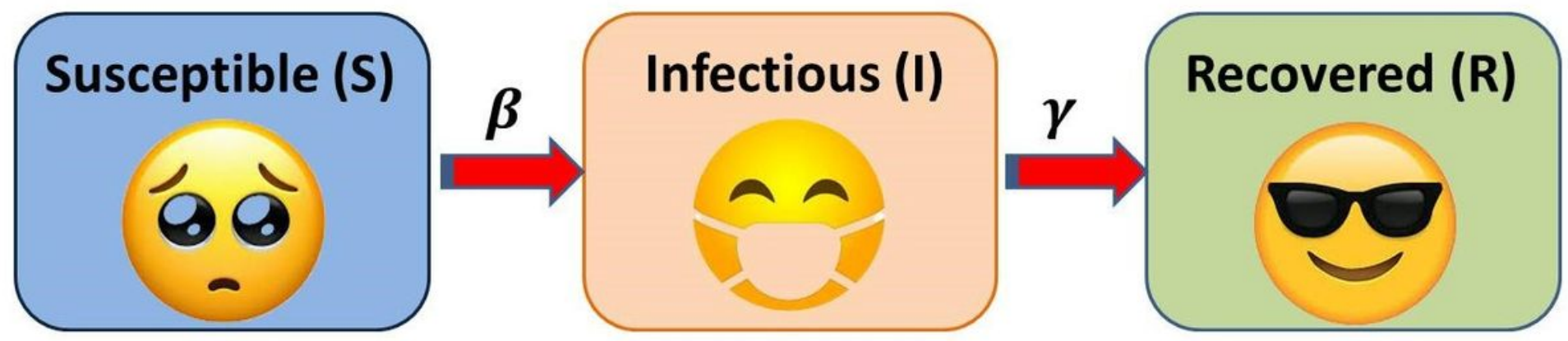

Figure 1

SIR model. 


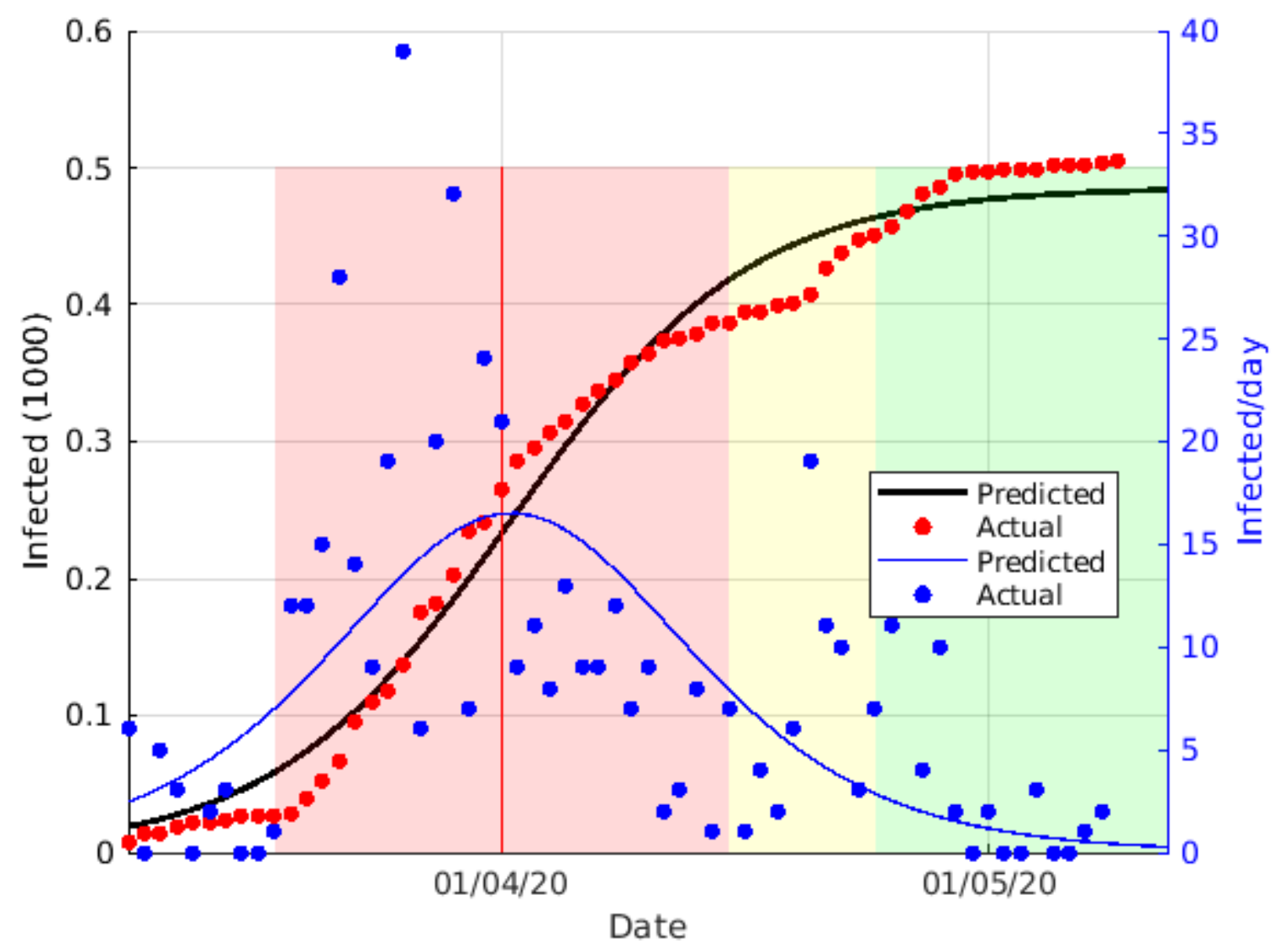

Figure 2

Logistic growth modelling of COVID-19 cases in Kerala. 


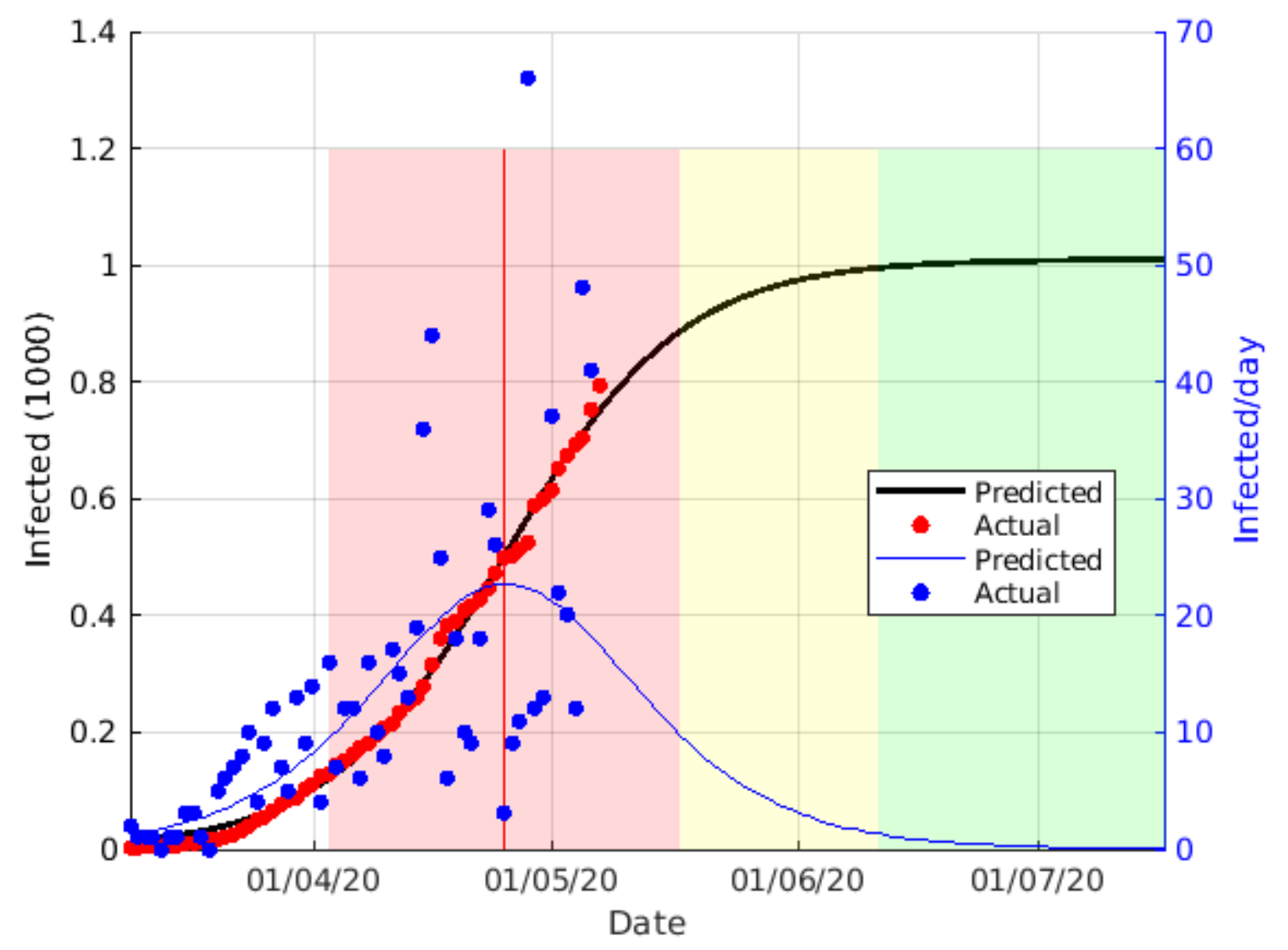

Figure 3

Logistic growth modelling of COVID-19 cases in Karnataka. 


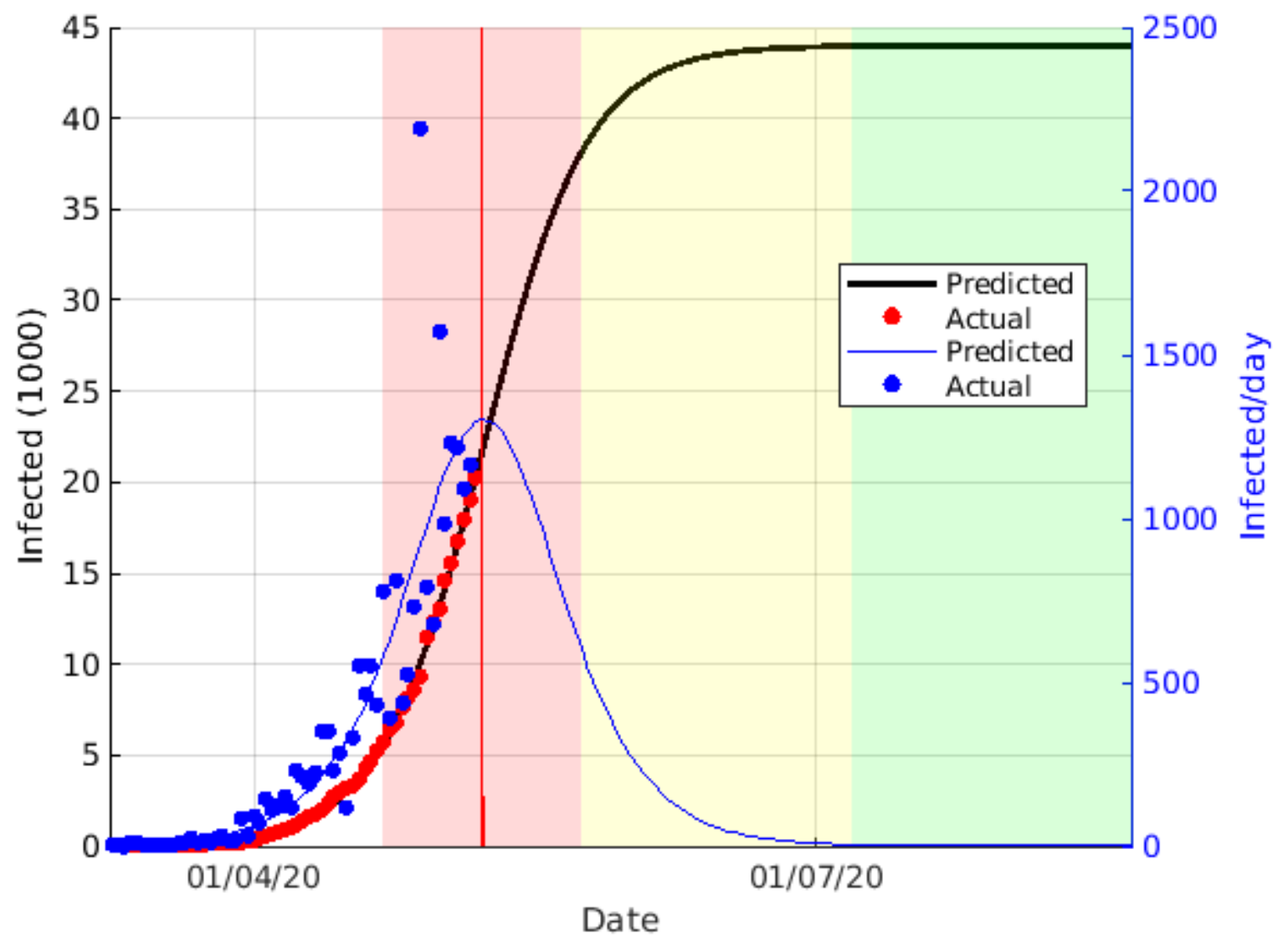

Figure 4

Logistic growth modelling of COVID-19 cases in Maharashtra. 


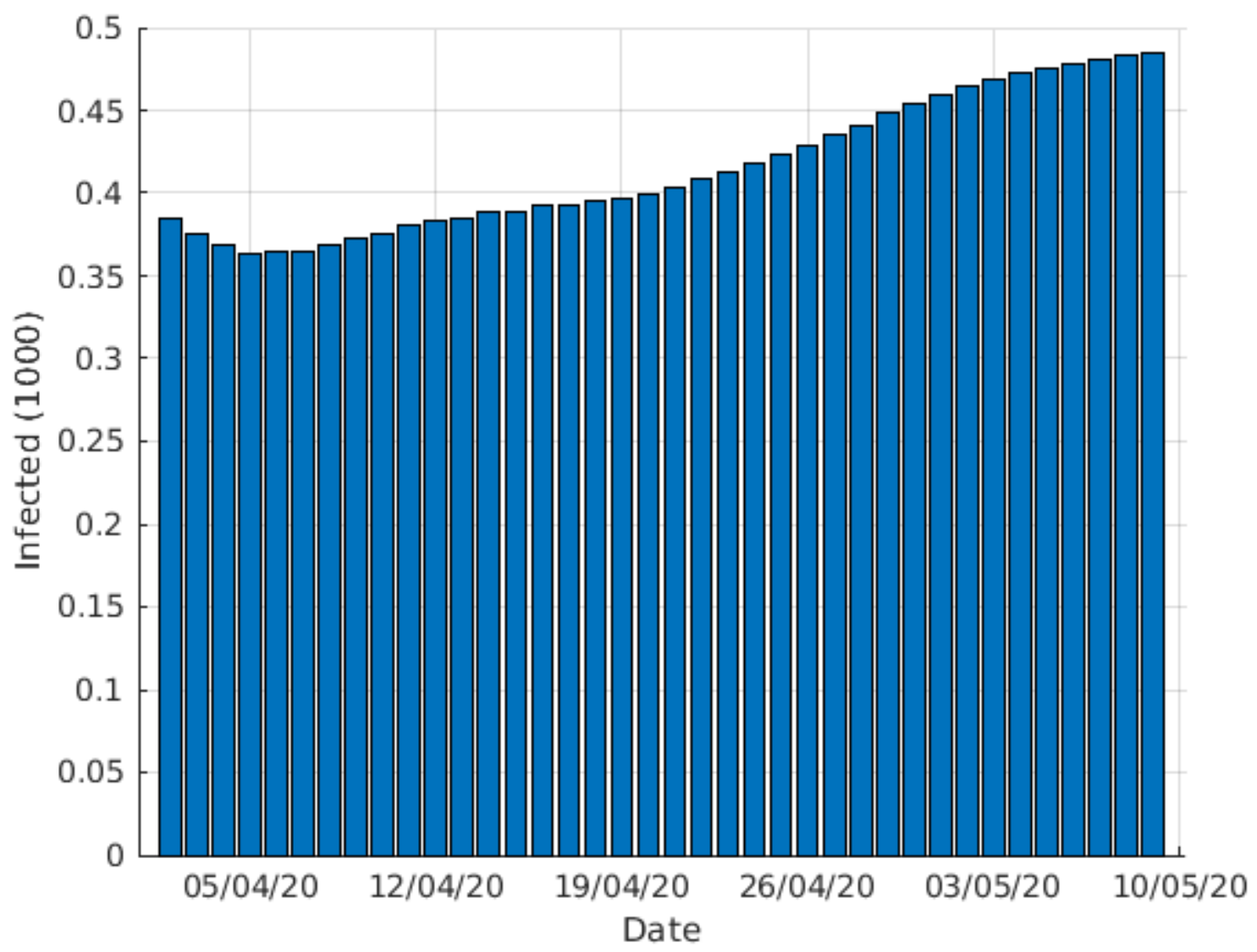

Figure 5

Daily estimated final size of COVID-19 cases in Kerala. 


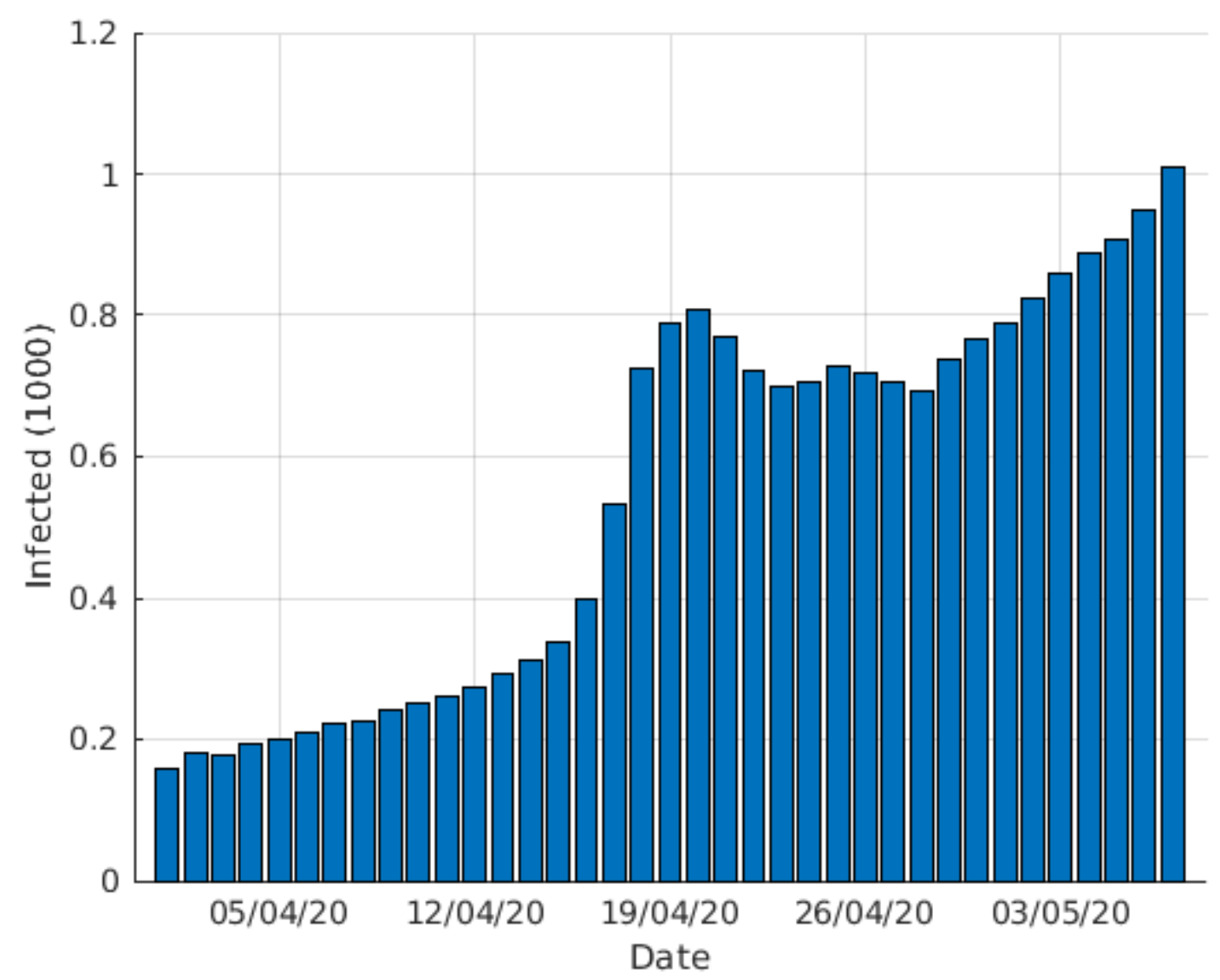

Figure 6

Daily estimated final size of COVID-19 cases in Karnataka. 


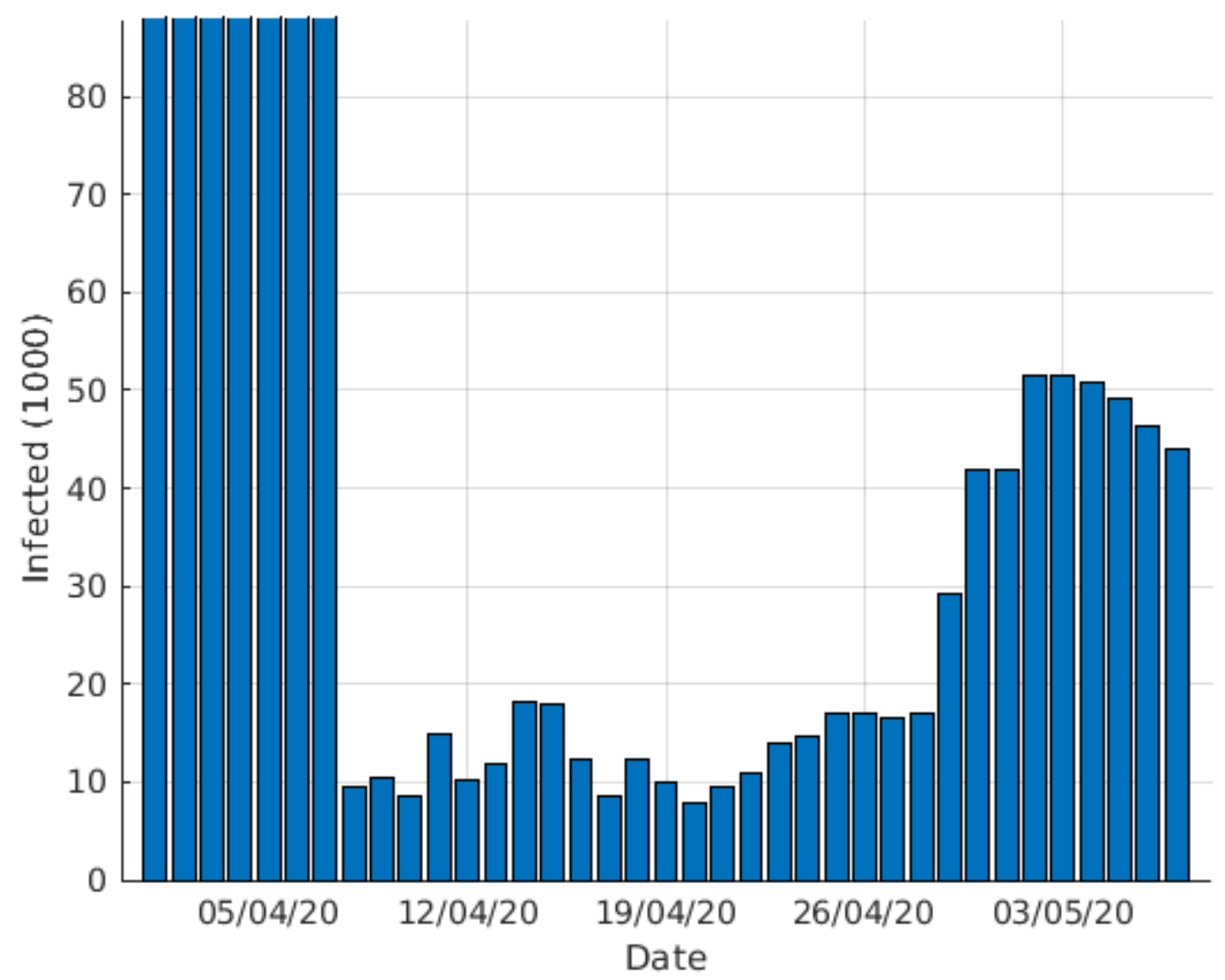

Figure 7

Daily estimated final size of COVID-19 cases in Maharashtra. 

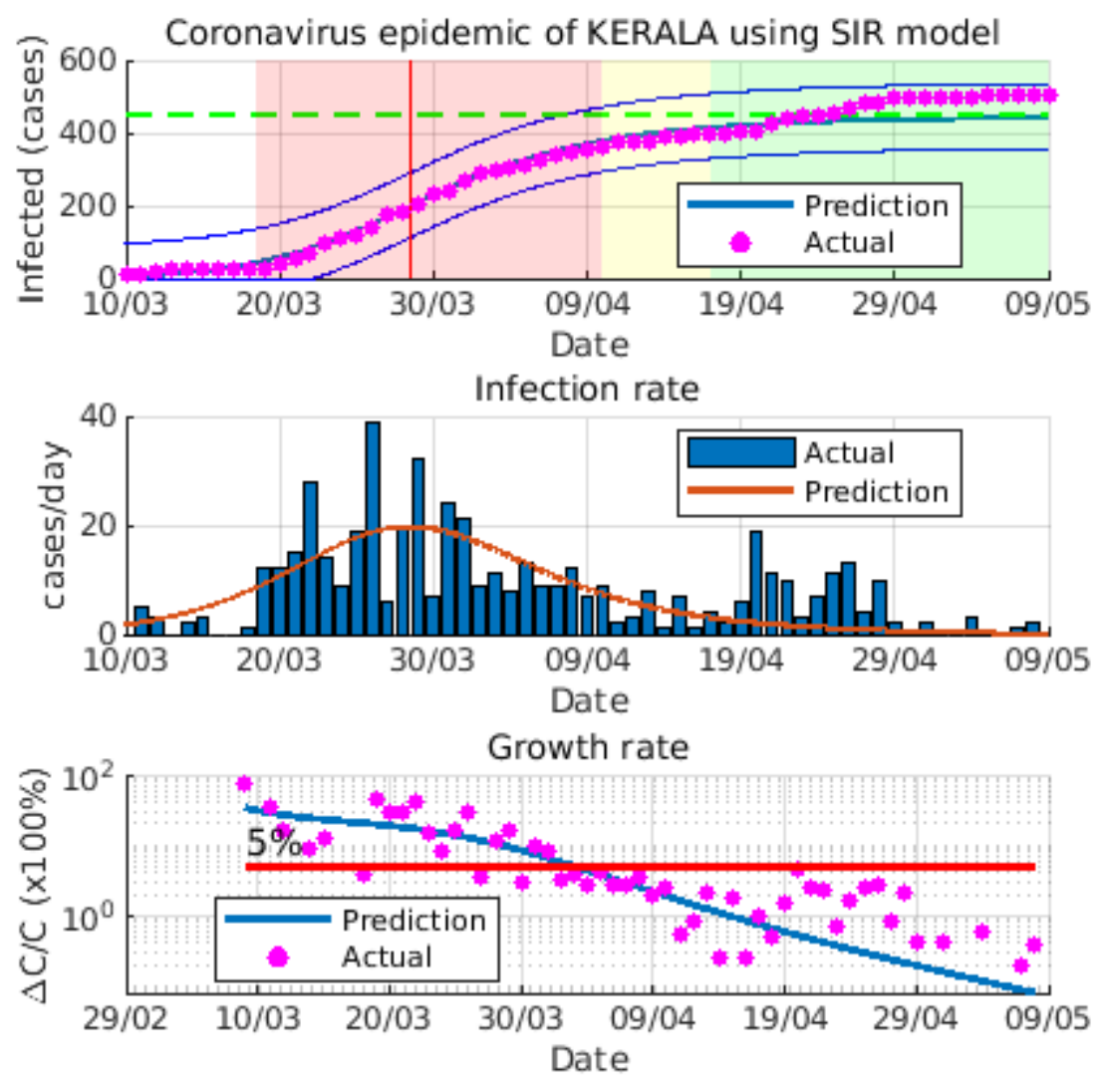

\section{Figure 8}

SIR modelling of COVID-19 cases in Kerala. 


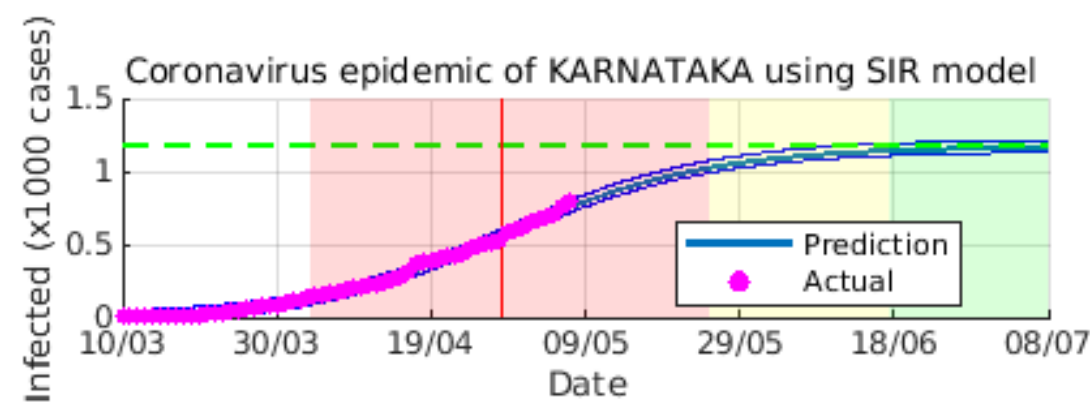

Infection rate

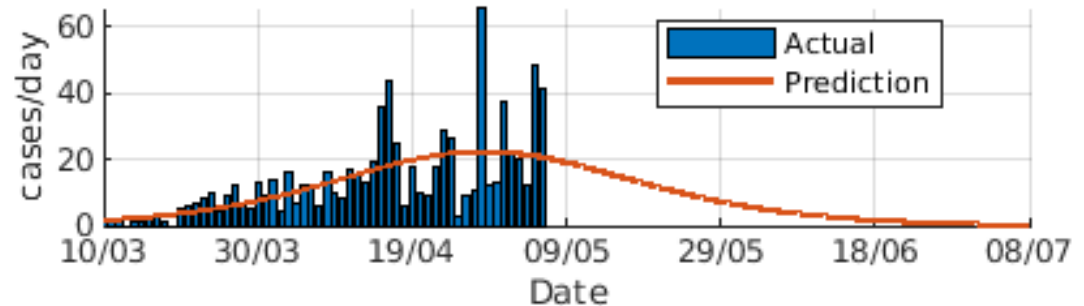

Growth rate

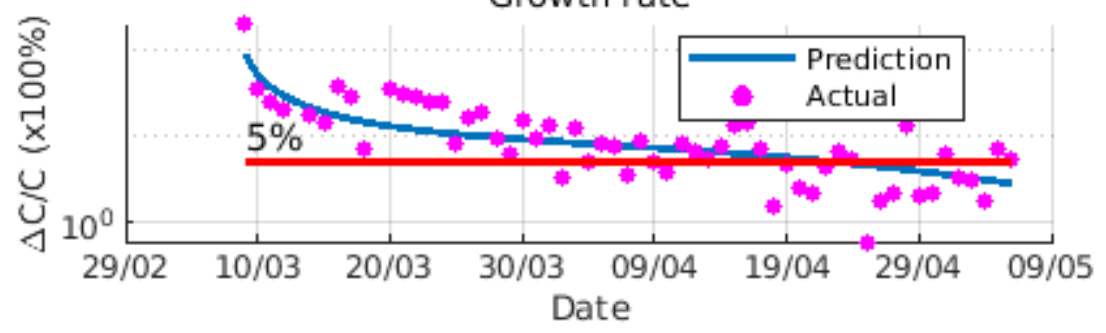

Figure 9

SIR modelling of COVID-19 cases in Karnataka. 

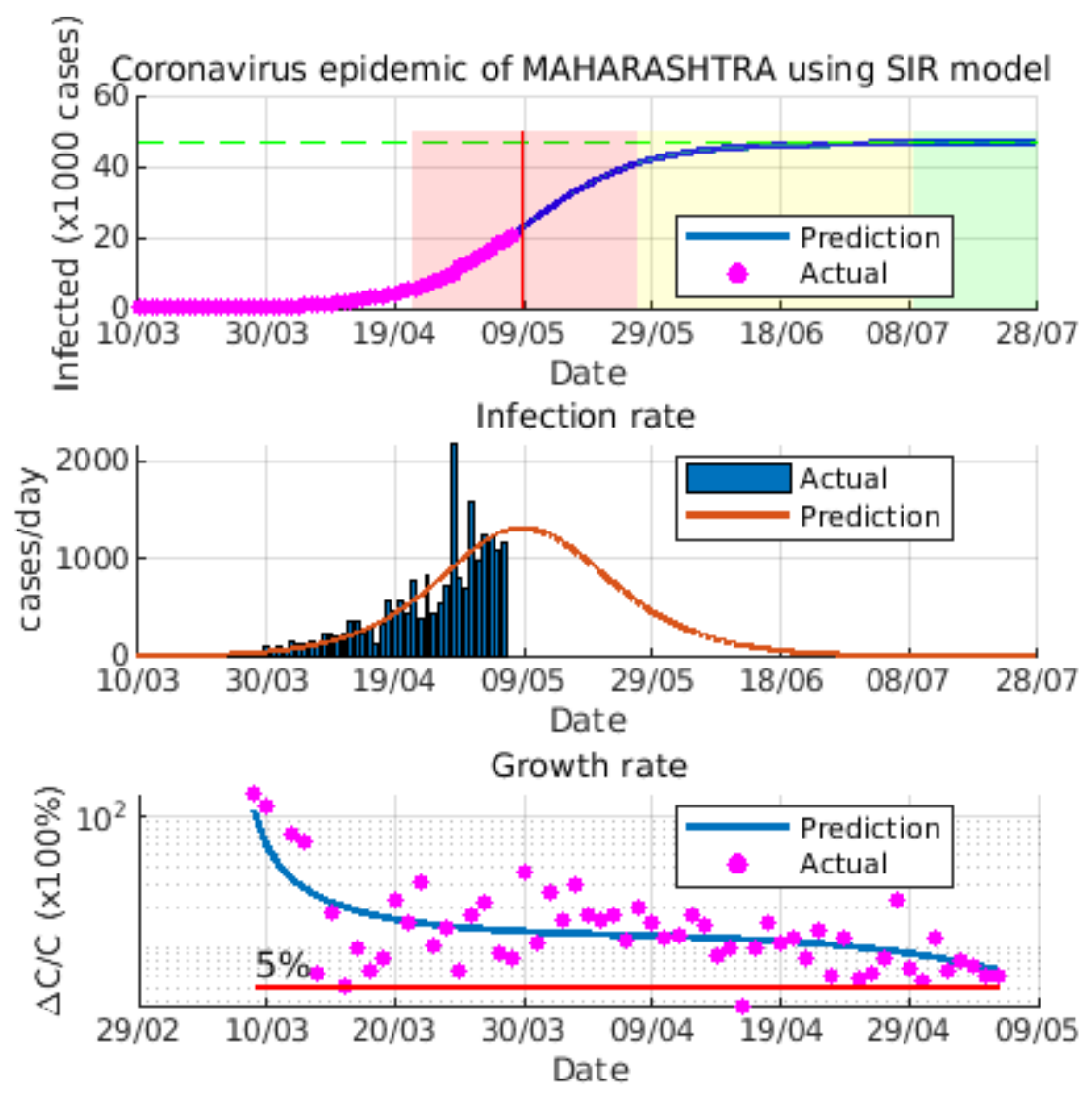

Figure 10

SIR modelling of COVID-19 cases in Maharashtra. 